SFB

Der Wert von Versorgungssicherheit mit Strom: Evidenz für deutsche Haushalte

Manuel Frondel, Stephan Sommer

Nr. 21/2017

$\infty$

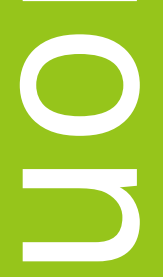

0

(1)
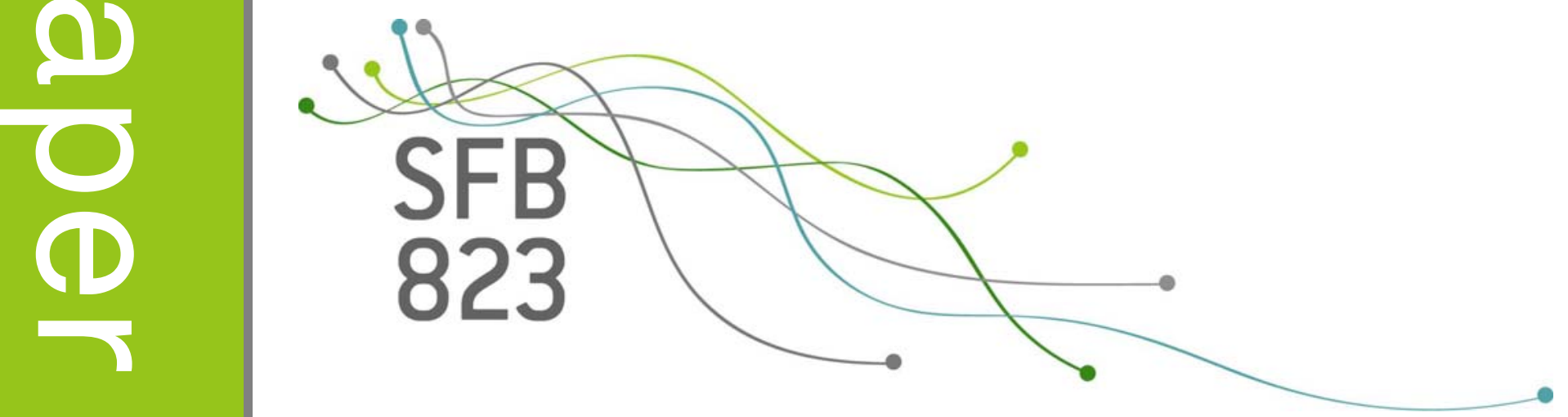



\title{
Der Wert von Versorgungssicherheit mit Strom: Evidenz für deutsche Haushalte
}

\author{
Manuel Frondel und Stephan Sommer
}

\section{Zusammenfassung}

Dieser Artikel untersucht auf Basis einer Befragung von mehr als 5.000 Haushaltsvorständen, wie viel sie für Versorgungssicherheit mit Strom zu zahlen bereit sind. Alternativ zur Zahlungsbereitschaft (willingness to pay, WTP) wird auch nach der Bereitschaft gefragt, gegen eine Entschädigungszahlung auf ein gewisses Maß an Versorgungssicherheit zu verzichten (willingness to accept, WTA). In Übereinstimmung mit zahlreichen empirischen Studien finden wir mittlere WTA-Werte, die deutlich über den mittleren WTP-Werten für die Vermeidung eines unangekündigten, vierstündigen Stromausfalls liegen. Den Grund für diese Diskrepanz sehen wir darin, dass die bekundeten Entschädigungsforderungen für den Verzicht auf Versorgungssicherheit tendenziell über dem tatsächlichen Wert liegen, der der Versorgungssicherheit mit Strom beigemessen wird, wohingegen die dafür bekundete Zahlungsbereitschaft tendenziell untertrieben wird.

Keywords: Zahlungsbereitschaft, bekundete Präferenzen, Energiewende.

JEL Codes: D12, H41, Q41.

Keywords: willingness to pay, willingness to accept, stated preferences.

Danksagung: Wir danken Carina Klebe für wertvolle Vorarbeiten, Marco Horvath für hilfreiche Kommentare sowie der Deutschen Forschungsgemeinschaft für die finanzielle Unterstützung im Rahmen des Sonderforschungsbereichs 823 "Statistische Modellierung Nichtlinearer Dynamischer Prozesse", Projekt A3, "Dynamische TechnologieModellierung”, und dem Bundesministerium für Bildung und Forschung (BMBF) für die finanzielle Unterstützung im Rahmen des Kopernikus-Projekts ENavi (Förderkennzeichen: 03SFK4B0) und des Projekts AKZEPTANZ (Förderkennzeichen: 01 UN 1203C) im Rahmen der Fördermaßnahme Umwelt- und gesellschaftsverträgliche Transformation des Energiesystems. 


\section{Einleitung}

Stromausfälle sind in Deutschland eher selten - besonders im europäischen Vergleich (CEER, 2016). Der Energiewende zum Trotz hat sich die Versorgungssicherheit mit Strom im vergangenen Jahrzehnt vermeintlich sogar verbessert. So ist die Dauer einer Versorgungsunterbrechung je Letztverbraucher und Kalenderjahr zwischen 2006 und 2015 gesunken, von durchschnittlich 21,53 auf 12,70 Minuten (BNetzA, 2017). Die Versorgungssicherheit mit Strom bewegt sich in Deutschland somit auf einem sehr hohen Niveau - trotz des im Jahr 2011 beschlossenen Ausstiegs aus der Atomkraft und dem hohen Tempo, mit dem der Ausbau der erneuerbaren Energietechnologien vorangetrieben wird. So stieg der Anteil von ,grünem“ Strom am Bruttostromverbrauch zwischen dem Jahr 2000, in dem das Erneuerbaren-Energien-Gesetz (EEG) eingeführt wurde, und dem Jahr 2016 von knapp 6\% auf rund 32\% (BMWi, 2017). Der Anteil des schwankend anfallenden grünen Stroms hat sich in diesem Zeitraum somit mehr als verfünffacht.

Aus mehreren Gründen könnte die Versorgungssicherheit mit Strom aber in absehbarer Zeit $\mathrm{zu}$ einem bedeutenden gesellschaftlichen Thema werden. Zum einen werden konventionelle Kraftwerke durch den sich stetig erhöhenden Anteil der Erneuerbaren und der infolgedessen stark gesunkenen Börsenstrompreise zunehmend unrentabler und gehen daher vermehrt vom Netz. Dies ist besonders im Süden Deutschlands, in dem durch die Abschaltung von Kernkraftwerken zeitweise ein Defizit an Kraftwerksleistung herrscht, nicht unproblematisch. Um diesem Problem zu begegnen, fordern Stimmen aus der Europäischen Kommission eine Aufteilung der bislang einheitlichen Preiszone in Deutschland in zwei Preiszonen, eine Zone für den Süden und eine zweite für den Norden (Welt, 2016).

Zum anderen sorgt die massive Zunahme der stark fluktuierenden Einspeisung von Windund Solarstrom für einen erschwerten Ausgleich von Angebot und Nachfrage (Römer et al., 2012). Zur Aufrechterhaltung der Netzstabilität müssen daher immer häufiger Windund Solarparks abgeschaltet werden. Die dafür anfallenden Entschädigungszahlungen an die Betreiber summierten sich im Jahr 2015 auf knapp 500 Mio. Euro. Gleichzeitig hat sich auch das Volumen sogenannter Redispatch-Maßnahmen erhöht. Die Kosten für diese von den Übertragungsnetzbetreibern angeordneten Eingriffe in den Fahrplan konventioneller Kraftwerke zur Vorbeugung von Leistungsüberlastungen im Stromnetz stiegen zwischen 2010 und 2015 von 13 auf rund 400 Mio. Euro (BDEW, 2017). 
Diese Kosten müssen die Stromverbraucher in Form von Netzentgelten mit ihren Stromrechnungen tragen. Die Bundesnetzagentur geht davon aus, dass sich die Kosten zur Aufrechterhaltung der Netzstabilität bis zum Jahr 2020 weiter erhöhen und auf 4 Mrd. Euro steigen könnten. Ohne derartige Maßnahmen könnte es zu Stromausfällen kommen, die ebenfalls mit erheblichen gesellschaftlichen Kosten verbunden sind. So schätzen Praktiknjo et al. (2011) und Röpke (2013) die Folgekosten (Value of Lost Load) für jede nicht gelieferte Kilowattstunde (kWh) Strom auf ca. 15 Euro, wobei diese Kosten regional sehr unterschiedlich ausfallen können (Growitsch et al., 2015; Wolf und Wenzel, 2016).

Die Versorgungssicherheit könnte zwar grundsätzlich durch Stromspeicher verbessert werden, da diese die Netzlast intertemporal ausgleichen und so helfen können, fluktuierende Energiequellen in das Stromsystem zu integrieren. Abgesehen von kleinen Batterien und Pumpspeicherkraftwerken sind viele Speichertechnologien aber noch weit von der Marktreife entfernt (Hochloff et al., 2014). Zudem ist der Neubau von Pumpspeicherkraftwerken einerseits topographisch und andererseits aufgrund von Akzeptanzproblemen und Nutzungskonflikten mit der Landwirtschaft sehr begrenzt (Stenzel et al., 2013).

Vor diesem Hintergrund untersucht dieser Beitrag die individuellen Präferenzen für die Versorgungssicherheit mit Strom auf Basis einer Befragung von mehr als 5.000 Haushalten. Mit Hilfe eines experimentellen Designs wird einerseits analysiert, wie viel Haushalte für die Versorgungssicherheit mit Strom zu zahlen bereit sind. Alternativ zur Zahlungsbereitschaft (willingness to pay, WTP) wird andererseits nach der Bereitschaft gefragt, gegen eine Entschädigungszahlung auf ein gewisses Maß an Versorgungssicherheit $\mathrm{zu}$ verzichten (willingness to accept, WTA). Obwohl nach ökonomischer Theorie bei gering ausfallenden Einkommenseffekten die maximale Zahlungsbereitschaft für den Konsum eines Gutes der minimalen Entschädigungsforderung im Falle des Verzichts auf das Gut entsprechen sollte, gibt es in der Literatur eine Vielzahl an Studien, die teils erhebliche Diskrepanzen zwischen diesen beiden Präferenzmaßen konstatieren - siehe z. B. Amigues et al. (2002), Guria et al. (2005), Knetsch (1990) sowie für einen Überblick Horowitz und McConnell (2002) oder Tunçel und Hammitt (2014).

In Übereinstimmung mit den Studien von Praktiknjo (2014) und Schubert et al. (2013), in denen das Verhältnis von WTA zu WTP für einen vierstündigen Stromausfall bei etwa 
$W T A / W T P=3$ liegt, finden wir mittlere WTA-Werte für Entschädigungsforderungen, die deutlich über den mittleren WTP-Werten für die Zahlungsbereitschaft für die Vermeidung eines unangekündigten, vierstündigen Stromausfalls liegen. Wie im folgenden Abschnitt erläutert wird, sehen wir den Grund für diese Diskrepanz darin, dass die bekundeten Entschädigungsforderungen für den Verzicht auf ein Gut aus strategischen Gründen tendenziell über dem tatsächlichen Wert liegen, der dem Gut beigemessen wird, wohingegen die dafür bekundete Zahlungsbereitschaft tendenziell untertrieben wird. Für Stromversorger, die ihren Kunden künftig Verträge anbieten möchten, in denen die heute noch so selbstverständliche - Versorgungssicherheit eine Rolle spielt, wäre es nach diesen Ergebnissen wenig ratsam, hohe Entschädigungszahlungen für die Möglichkeit anzubieten, unangekündigte Stromabschaltungen vornehmen zu dürfen.

Der folgende Abschnitt bietet eine kurze Zusammenfassung der in der Literatur angeführten Gründe für die Diskrepanz zwischen WTA und WTP. Abschnitt III stellt die Erhebung und Datenbasis vor, Abschnitt IV das Design des Experiments. In den Abschnitten V und VI werden die Ergebnisse der Analyse präsentiert. Der letzte Abschnitt fasst zusammen und zieht Schlussfolgerungen.

\section{Die Lücke zwischen WTA und WTP}

Die Standardannahmen der ökonomischen Theorie implizieren, dass bei kleinen Einkommenseffekten der Unterschied zwischen der maximalen Zahlungsbereitschaft für ein Gut und der minimalen Entschädigungsforderung für den Verzicht auf das Gut vernachlässigbar sein sollte (Willig, 1976). Diese Implikation steht jedoch in scharfem Kontrast zu dem empirischen Befund der sogenannten WTA-WTP-Lücke (z. B. Kahneman et al., 1990): die Verkaufspreise (WTA) für ein Gut fallen typischerweise signifikant höher aus als die Kaufpreise (WTP). Thaler (1980) fand zum Beispiel, dass die minimale Entschädigungsforderung für die Akzeptanz eines Risikos von 0,1\%, einen plötzlichen Tod zu erleiden, mindestens 10 Mal höher ausfiel als der Betrag, den die Probanden für die Vermeidung dieses Risikos zu zahlen bereit waren.

In der Literatur werden unterschiedliche Ursachen für die WTA-WTP-Lücke angeführt. So vertreten Kahneman et al. (1990) sowie Koetse und Brouwer (2016) die Auffassung, dass diese Lücke genuiner Natur ist und auf den sogenannten Endowment-Effekt zurückgeht. Damit bezeichnete Thaler (1980) die Wertsteigerung, die Individuen einem Gut beimessen, sobald dieses in ihren Besitz übergegangen ist. Eine damit 
zusammenhängende Erklärung ist der sogenannte Status-Quo-Bias: Individuen neigen dazu, den gegenwärtigen Zustand gegenüber Veränderungen übermäßig zu bevorzugen und sind im Allgemeinen wenig bereit, einen einmal erreichten Zustand aufzugeben (Mandler, 2004). Beide Effekte - der Endowment Effekt und der Status-Quo-Bias stellen eine Manifestation von Verlustaversion dar, nach der Verluste erheblich höher bewertet werden als Gewinne in entsprechender Höhe.

Hanemann (1991) führt als alternative Erklärung den Grad der Substituierbarkeit eines Gutes an. Ist ein Gut schwer substituierbar, ist laut Hanemann (1991) mit einer großen WTA-WTP-Diskrepanz zu rechnen. Ist hingegen ein Gut leicht substituierbar ist, sollte die Differenz zwischen den WTA- und WTP-Werten klein sein. Demnach sollte man eine Konvergenz der WTA- und WTP-Werte in Abhängigkeit des Grades der Substituierbarkeit erwarten können. Shogren et al. (1994) testen und bestätigen diese Hypothese mit Hilfe eines experimentellen Designs, bei dem einerseits die WTA- und WTP-Werte für leicht substituierbare am Markt gehandelte Güter (Kaffeetassen und Schokoriegel) und andererseits für ein nicht am Markt handelbares, schwer substituierbares Gut (verringerte Gesundheitsrisiken) ermittelt werden.

Auch die im Folgenden dargestellte Diskrepanz zwischen den Entschädigungsforderungen bei Akzeptanz eines unangekündigten, vierstündigen Stromausfalls und der Zahlungsbereitschaft für die Vermeidung eines ebenso gearteten Stromausfalls könnte durch die Hypothese von Hanemann (1991) erklärt werden, da der Stromverbrauch von Haushalten in der Regel recht unelastisch und Strom schwer zu substituieren ist. In einer Erhebung hypothetischer WTA- und WTP-Werte, wie sie hier durchgeführt wird, erscheint eine andere Erklärung jedoch naheliegender.

Diese Erklärung beruht darauf, dass in hypothetischen Befragungen nicht unbedingt die wahre maximale Zahlungsbereitschaft bzw. die tatsächliche Mindestforderung für eine Entschädigungszahlung bekundet werden. Aufgrund des hypothetischen Charakters der Abfrage ist es keineswegs abwegig, den Probanden strategisches Verhalten zu unterstellen. So könnten die Probanden einerseits Forderungen für eine Entschädigungszahlung bekunden, die über ihrer tatsächlichen Mindestforderung liegen, und andererseits Zahlungsbereitschaften signalisieren, die sich unterhalb ihrer jeweiligen wahren Zahlungsbereitschaft befinden. 
Dies steht in Einklang mit Carson und Groves (2007), die zeigen, dass es aus der Sicht eines Teilnehmers einer Erhebung optimal ist, bei privaten Gütern eine geringere Zahlungsbereitschaft anzugeben, als es den tatsächlichen Präferenzen entspricht. Denn wenn die Preise eines Gutes auf Basis von erhobenen Zahlungsbereitschaften festgesetzt werden, werden sie umso niedriger ausfallen, je niedriger die durchschnittliche Zahlungsbereitschaft der Abnehmer ausfällt (vgl. z. B. Loomis et al., 2000). Nehmen folglich Haushalte Versorgungssicherheit als ein privates Gut wahr, wäre es für sie strategisch optimal, nicht ihre wahre Zahlungsbereitschaft zu offenbaren und stattdessen geringere Zahlungsbereitschaften zu bekunden. Analog wäre es auch strategisch optimal, ihre Entschädigungsforderungen zu übertreiben (Groothuis und Miller, 1994). Tatsächlich ergibt sich aus einer Vielzahl an Studien, dass in hypothetischen Befragungen der WTAWert übertrieben wird (z. B. List und Gallett, 2001; Nape et al., 2003). ${ }^{1}$

Die in diesem Beitrag ermittelte WTA-WTP-Lücke könnte somit die Folge möglicher Übertreibungen bei den bekundeten WTA-Werten und potentieller Untertreibungen bei den WTP-Werten sein. Gemäß dieser Erklärung würde die WTA-WTP-Lücke nur bei hypothetischen Befragungen existieren und bei der Erhebung von offenbarten Präferenzen verschwinden. Dies stünde im Einklang mit Plott und Zeiler (2005), die die Existenz der WTA-WTP-Lücke bezweifeln und diese als das Resultat von Experimenten mit schlechtem Design darstellen. ${ }^{2}$

Tatsächlich aber lässt sich unsere Erklärung auch für die Erhebung von offenbarten Präferenzen aufrechterhalten, wenn unterstellt wird, dass die Probanden ihre eigenen Zahlungsbereitschaften bzw. Entschädigungsforderungen nicht perfekt kennen, sondern nur jeweils in einer gewissen Bandbreite. Gerade im Fall von Versorgungssicherheit ist davon auszugehen, dass es den Haushalten mangels Erfahrung schwerfällt, einen genauen Wert für die Vermeidung eines Stromausfalls einer gewissen Dauer anzugeben. Die Haushalte dürften allenfalls in der Lage sein, eine grobe Bandbreite dafür anzugeben. Dann wäre es rational, wenn die Probanden die Obergrenze des Intervalls als ihre Mindestforderung nennen würden bzw. die Untergrenze der Bandbreite als ihre maximale Zahlungsbereitschaft.

\footnotetext{
${ }^{1}$ Dies gilt häufig auch für die Zahlungsbereitschaft (WTP) für nicht am Markt gehandelte Güter, was als Hypothetical Bias bezeichnet wird (siehe z. B. Blumenschein et al., 2008; Harrison, 2006).

2 Dieses Argument hat zu einer Debatte zwischen Isoni et al. (2011) und Plott und Zeiler (2011) im American Economic Review geführt.
} 
III. Daten

Die diesem Beitrag zugrundeliegende Datenbasis wurde in einer gemeinschaftlichen Erhebung des RWI und des Marktforschungsinstituts forsa gewonnen. Diese Erhebung, die im Rahmen des vom Bundesministerium für Bildung und Forschung (BMBF) geförderten Projektes AKZEPTANZ durchgeführt wurde, fand zwischen dem 23. Dezember 2015 und dem 19. Februar 2016 statt. Befragt wurden über 5.000 Haushaltsvorstände des forsa-Haushaltpanels zu unterschiedlichen Aspekten der Energiewende. Während Panel-Haushalte ohne Internetanschluss mit Hilfe des Fernsehers teilnehmen konnten, füllte die große Mehrheit den eigens für diese Befragung konzipierten Fragebogen via Internet aus. Bei vollständiger Beantwortung des Fragebogens konnten die Teilnehmer Bonuspunkte erwerben, die sie ähnlich wie bei einem Payback-System in Prämien eintauschen können.

Die Haushalte des forsa-Haushaltspanels verfügen über Erfahrung mit dieser Art von Befragungen und sind repräsentativ für die deutschsprachige Bevölkerung ab dem Alter von 14 Jahren. Die regionale Verteilung der Stichproben-Haushalte entspricht recht genau der Verteilung der Grundgesamtheit der Haushalte in Deutschland, wie sie der Mikrozensus für das Jahr 2015 ausweist. Für die Zwecke dieser Erhebung wurden die Haushaltsvorstände befragt. Diese treffen per definitionem üblicherweise die finanziellen Entscheidungen, die den gesamten Haushalt betreffen. Aus diesem Grund ist der Anteil der Frauen unter den Antwortenden mit 37,7\% niedriger als in der Bevölkerung (Tabelle $1)$.

Unsere Stichprobe ist darüber hinaus nicht repräsentativ in Bezug auf das Merkmal Bildung und weist den sogenannten Bildungsbias auf, der für Erhebungen in Deutschland typisch ist (Andor et al., 2014). So verfügt mit 33,1\% ein hoher Anteil der Haushaltsvorstände über einen Hochschulabschluss. In der deutschen Bevölkerung liegt dieser Anteil bei lediglich 20,4\% (siehe Tabelle A1 im Anhang). Die im Folgenden dargestellten Werte für Versorgungssicherheit gelten daher allein für die Stichprobe, nicht aber für die Bevölkerung insgesamt.

In Bezug auf die Versorgungssicherheit mit Strom wurden verschiedene Fragen gestellt, etwa nach der geschätzten Zahl der Stromausfälle, die der Haushalt in den vergangenen fünf Jahren erlebt hat. Knapp zwei Drittel der Haushalte gab an, in den vergangenen fünf Jahren keinen Stromausfall mit einer Mindestdauer von 30 Minuten erlebt zu haben. 
Tabelle 1: Deskriptive Statistiken

\begin{tabular}{|c|c|c|c|}
\hline Variable & Erläuterung & $\begin{array}{r}\text { arithm. } \\
\text { Mittel }\end{array}$ & $\begin{array}{l}\text { Std. } \\
\text { Abw. }\end{array}$ \\
\hline Alter & Alter der Befragten & 55,11 & 13,4 \\
\hline Frau & Dummy: 1, falls der Haushaltsvorstand weiblich ist & 0,377 & - \\
\hline Kinder im Haushalt & Dummy: 1, falls Kinder im Haushalt leben & 0,442 & - \\
\hline \multirow[t]{2}{*}{ Ostdeutschland } & Dummy: 1 , falls der Haushalt in & & \\
\hline & Ostdeutschland wohnt & 0,198 & - \\
\hline Hochschulabschluss & $\begin{array}{l}\text { Dummy: 1, falls der Haushaltsvorstand einen } \\
\text { (Fach-) Hochschulabschluss hat }\end{array}$ & 0,331 & - \\
\hline Grünen-Wähler & $\begin{array}{l}\text { Dummy: 1, falls der Haushaltsvorstand dazu neigt, } \\
\text { die Partei der Grünen zu wählen }\end{array}$ & 0,102 & - \\
\hline Einkommen & Monatliches Haushaltsnettoeinkommen in $€$ & 2.893 & 1.371 \\
\hline Erwerbstätig & Dummy: 1 , falls der Haushaltsvorstand erwerbstätig ist & 0,560 & - \\
\hline Eigentümer & Dummy: 1, falls der Haushalt in Eigentum wohnt & 0,608 & - \\
\hline Grünstrom & Dummy: 1, falls der Haushalt Grünstrom bezieht & 0,282 & - \\
\hline \multirow{2}{*}{ Erneuerbaren-Anlage } & Dummy: 1 , falls der Haushalt eine & & \\
\hline & Erneuerbaren-Anlage betreibt & 0,125 & - \\
\hline \multirow[t]{2}{*}{ Jährlicher Stromverbrauch } & Vom Haushaltsvorstand geschätzter jährlicher & & \\
\hline & Stromverbrauch in $\mathrm{kWh}$ & 3.786 & 4.285 \\
\hline \multirow[t]{2}{*}{ Finanzielle Konsequenzen } & Dummy: 1, falls der Haushaltsvorstand finanzielle & & \\
\hline & Konsequenzen durch die Befragung erwartet & 0,292 & - \\
\hline \multirow[t]{2}{*}{ Politische Konsequenzen } & Dummy: 1, falls der Haushaltsvorstand politische & & \\
\hline & Konsequenzen durch die Befragung erwartet & 0,728 & \\
\hline Stromausfall erlebt & $\begin{array}{l}\text { Dummy: } 1 \text {, falls in den vergangenen } 5 \text { Jahren } \\
\text { ein mehr als } 30 \text {-minütiger Stromausfall erlebt wurde }\end{array}$ & 0,363 & - \\
\hline \multirow[t]{2}{*}{ \# erlebte Stromausfälle } & Anzahl der in den vergangenen & & \\
\hline & 5 Jahren erlebten Stromausfälle & 3,07 & 3,84 \\
\hline Länge des Stromausfalls & $\begin{array}{l}\text { Dauer des längsten Stromausfalls der in } \\
\text { den letzten } 5 \text { Jahren erlebten Stromausfälle in Minuten }\end{array}$ & 109,2 & 281,6 \\
\hline
\end{tabular}

Zur Ermittlung der Zahlungsbereitschaft für Versorgungssicherheit mit Strom bedienen wir uns einer hypothetischen Befragung. Hierzu gibt es praktisch keine Alternative, denn es wäre nur schwerlich möglich, die tatsächlichen Präferenzen zu ermitteln. Dazu müsste den Haushalten in zufälliger Weise der Strom abgestellt werden und ihnen zudem die Möglichkeit eingeräumt werden, sich davon entweder freikaufen zu können (WTP) oder aber Entschädigungen zu erhalten (WTA).

\section{Experimentelles Design}

Zur Ermittlung des Wertes, den die Bürger der Versorgungssicherheit mit Strom beimessen, wurde ein Experiment durchgeführt, bei dem die Teilnehmer randomisiert in zwei Gruppen eingeteilt wurden: entweder in die WTP-Gruppe, in der die Teilnehmer ihre Zahlungsbereitschaft für Versorgungssicherheit äußern sollten, oder in die WTA-Gruppe, in der die Teilnehmer nach ihrer Bereitschaft gefragt wurden, eine gewisse Entschädigung für einen Stromausfall zu akzeptieren. Alle Teilnehmer wurden zuvor darüber informiert, 
dass es aus Gründen der Versorgungssicherheit sinnvoll sein könnte, einzelnen Haushalten den Strom abzustellen.

Sodann wurden die Teilnehmer der WTA-Gruppe nach ihrer Bereitschaft gefragt, eine monatliche Entschädigung von x Euro zu akzeptieren, wenn ihnen der Netzbetreiber einmal im Jahr für maximal vier Stunden unangekündigt den Strom abstellen darf. Der konkrete Betrag der monatlichen Entschädigung von $\mathrm{x}=3,6$ oder 9 Euro wurde den Haushalten randomisiert per Zufallsgenerator zugewiesen. Die Teilnehmer der WTPGruppe wurden hingegen nach ihrer Zahlungsbereitschaft zur Vermeidung einer gleich gearteten Abschaltung des Stroms gefragt. Dazu wurden ihnen randomisiert einer von drei Beträgen für monatliche Zahlungen von 3, 6 oder 9 Euro genannt, welchen sie akzeptieren oder ablehnen konnten. (Alle Fragen des Entscheidungsexperiments sind im Detail im Anhang dargestellt.)

Diese Form von Experiment, im Angelsächsischen mit „single binary choices“ bezeichnet, wird unter anderem sowohl vom NOAA-Panel ${ }^{3}$ (Arrow et al., 1993) als auch von Carson und Groves (2007) empfohlen, weil sie unter bestimmten Umständen anreizkompatibel sei; mithin würden Teilnehmer ihre wahren Präferenzen äußern. Eine wesentliche Annahme dafür ist, dass die Teilnehmer eines Experimentes glauben, damit könnten zumindest im Prinzip - finanzielle Konsequenzen verbunden sein (Carson, Groves, 2007: 188). Dies wäre in diesem Fall erfüllt, wenn die Teilnehmer glauben, dass Stromversorger künftig Stromlieferverträge anbieten, in denen das Niveau der Versorgungssicherheit explizit eine Rolle spielt.

Alternativ zur zufälligen Vorgabe eines von drei Geld-Beträgen hätten wir die Studienteilnehmer bitten können, einen von ihnen selbst gewählten Betrag für ihre Zahlungsbereitschaft bzw. ihre Bereitschaft zur Akzeptanz von Entschädigungszahlungen zu nennen. Allerdings haben sich solche sogenannten Contingent-Valuation-Designs als eher ungeeignet erwiesen, da Probanden oftmals Schwierigkeiten damit hatten, eine konkrete Zahl zur Bewertung eines öffentlichen oder abstrakten Guts zu nennen (Carson, Groves, 2007). Dies gilt aufgrund der relativ geringen Erfahrung der Haushalte mit Stromausfällen besonders im Fall der Versorgungssicherheit mit Strom.

\footnotetext{
3 Für das NOAA-Panel on Contingent Valuation (CV) der National Oceanic and Atmospheric Administration (NOAA) wurde im Jahr 1992 ein Forum von Sozialwissenschaftlern unter Führung der beiden Nobelpreisträger Kenneth Arrow und Robert Solow ernannt, um die Validität von CV-Maßen zu bewerten.
} 
Eine besondere Schwierigkeit besteht bei hypothetischen Befragungen darin, dass nicht unbedingt die wahre maximale Zahlungsbereitschaft bzw. die tatsächliche Mindestforderung für eine Entschädigungszahlung bekundet wird. Sowohl Carson und Groves (2007) als auch Herriges et al. (2010) argumentieren, dass Haushalte ihre wahre Zahlungsbereitschaft nur dann angeben, wenn sie davon ausgehen, dass sie durch die Teilnahme an der Erhebung politische Entscheidungen beeinflussen können und dass finanzielle Konsequenzen auf sie zukommen. Tatsächlich zeigen Vossler et al. (2012) in einer empirischen Studie, dass die Zahlungsbereitschaft sowohl sinkt, wenn Haushalte erwarten, dass sie politische Ergebnisse mitgestalten können, als auch dann, wenn Haushalte glauben, dass ihre Angabe finanziell bindend sei. Auf den Erkenntnissen von Vossler et al. (2012) basierend wurde in der Erhebung ein entsprechender KorrekturAnsatz verfolgt.

\section{Deskriptive Ergebnisse}

Als Erstes wird untersucht, ob die im Abschnitt III beschriebene Randomisierung erfolgreich war. Tatsächlich lassen die in Tabelle 2 dargestellten t-Statistiken keine statistisch signifikanten Abweichungen in den arithmetischen Mittelwerten der erklärenden Variablen zwischen der WTA- und der WTP-Gruppe erkennen. Basierend auf diesem Vergleich kann im Folgenden davon ausgegangen werden, dass die beiden Gruppen ausreichend randomisiert und die empirischen Ergebnisse nicht das Resultat unterschiedlicher Verteilungen der erklärenden Variablen sind.

Die deskriptiven Ergebnisse des Experiments zeigen (Tabelle 3), dass mit einem Anteil von $16,3 \%$ nur ungefähr jeder sechste Haushaltsvorstand bereit wäre, einen unangekündigten, vierstündigen Stromausfall im Jahr für eine monatliche Entschädigung von 3 Euro zu akzeptieren. Bei einer monatlichen Entschädigung von 6 Euro fällt die Akzeptanzrate mit 19,2\% geringfügig, aber nicht statistisch signifikant, höher aus. Mit einem Anteil von 23,7\% ist die Zustimmung zu einer monatlichen Entschädigung von 9 Euro jedoch statistisch signifikant höher als im Falle einer geringeren Entschädigungszahlung von nur 3 Euro.

Offenbar steigt die Akzeptanz für Stromausfälle mit der Höhe der Entschädigungen tendenziell an. Hingegen sinkt die Zahlungsbereitschaft für die Vermeidung eines unangekündigten, vierstündigen Stromausfalls pro Jahr mit den vorgegebenen Zahlungsbeträgen: Die Anteile an Haushalten, die bereit sind, dafür eine Zahlung von 
monatlich 3, 6 oder 9 Euro zu leisten, verringern sich von 23,2\% auf 15,0\% bzw. 11,8\%. Kurzum: Im Einklang mit der ökonomischen Theorie nimmt die Akzeptanz von Entschädigungszahlungen mit höheren Geldbeträgen zu, die Zahlungsbereitschaft nimmt hingegen $a b$.

Tabelle 2: Vergleich der arithmetischen Mittel der Charakteristika der WTA- und WTP-Gruppe

\begin{tabular}{lccc}
\hline Variable & $\begin{array}{c}\text { WTA } \\
\text { Gruppe }\end{array}$ & $\begin{array}{c}\text { WTP } \\
\text { Gruppe }\end{array}$ & t -Statistiken \\
\hline Alter & 55,31 & 54,91 & 1,13 \\
Frau & 0,370 & 0,384 & $-1,10$ \\
Kinder im Haushalt & 0,417 & 0,432 & 1,13 \\
Ostdeutschland & 0,197 & 0,200 & $-0,27$ \\
Hochschulabschluss & 0,333 & 0,328 & 0,40 \\
Grünen-Wähler & 0,104 & 0,099 & 0,48 \\
Einkommen & 2.908 & 2.879 & 0,75 \\
Erwerbstätig & 0,560 & 0,560 & 0,04 \\
Eigentümer & 0,604 & 0,612 & $-0,60$ \\
Grünstrom & 0,278 & 0,285 & $-0,60$ \\
Erneuerbaren-Anlage & 0,133 & 0,117 & 1,81 \\
Stromheizung & 0,031 & 0,036 & $-1,03$ \\
Jährlicher Stromverbrauch & $3.745,2$ & $3.826,5$ & $-0,59$ \\
Finanzielle Konsequenzen & 0,281 & 0,303 & $-1,72$ \\
Stromausfall erlebt & 0,369 & 0,356 & 0,96 \\
\# erlebte Stromausfälle & 3,03 & 3,10 & $-0,46$ \\
Länge des Stromausfalls & 113,0 & 105,3 & 0,68 \\
\hline Abbrecher-Quote & 0,035 & 0,043 & $-1,55$ \\
Anzahl an Beobachtungen: & 2.775 & 2.865 & - \\
\hline Statisiken geben die Unts
\end{tabular}

Anmerkung: Die t-Statistiken geben die Unterschiede im arithmetischen Mittel der jeweiligen Variablen zwischen der WTA- und der WTP-Gruppe an.

Tabelle 3: Anteile der Ja-Antworten zu den Fragen nach der Akzeptanz einer monatlichen Entschädigung (WTA) bzw. Zahlung (WTP) für die Tolerierung bzw. Vermeidung eines unangekündigten vierstündigen Stromausfalls pro Jahr

\begin{tabular}{ccccccc}
\hline & \multicolumn{2}{c}{ Gesamte Stichprobe } & \multicolumn{2}{c}{$\begin{array}{l}\text { Befragte, die finanzielle } \\
\text { Konsequenzen erwarten }\end{array}$} & \multicolumn{2}{c}{$\begin{array}{c}\text { Befragte, die politische } \\
\text { Konsequenzen erwarten }\end{array}$} \\
\hline \multirow{2}{*}{3 Euro } & 0,163 & 0,232 & 0,221 & 0,344 & 0,155 & 0,245 \\
& - & - & - & - & - & - \\
6TA Euro & 0,192 & 0,150 & 0,281 & 0,177 & 0,191 & 0,163 \\
& $(1,53)$ & $\left(-4,31^{* *}\right)$ & $(1,51)$ & $\left(-4,25^{* *}\right)$ & $(1,65)$ & $\left(-3,52^{* *}\right)$ \\
9 Euro & 0,237 & 0,118 & 0,362 & 0,143 & 0,258 & 0,125 \\
& $\left(3,72^{* *}\right)$ & $\left(-6,20^{* *}\right)$ & $\left(3,30^{* *}\right)$ & $\left(-5,35^{* *}\right)$ & $\left(4,35^{* *}\right)$ & $\left(-5,36^{* *}\right)$ \\
\hline
\end{tabular}

Anmerkung: t-Statistiken für Unterschiede im Mittelwert zwischen der 3-Euro-Gruppe und der 6 bzw. 9Euro-Gruppe sind in Klammern angegeben. ** bzw. * zeigen statistische Signifikanz auf dem 1\%- bzw. 5\%Niveau an

Um die in hypothetischen Abfragen von Zahlungsbereitschaften häufig auftretenden Abweichungen der bekundeten Werte von den in einem realen Marktumfeld offenbarten Präferenzen zu ermitteln, wurde hier ein Korrektur-Ansatz verfolgt, der auf Erkenntnissen von Carson und Groves (2007) und Vossler et al. (2012) beruht. Demnach offenbaren die Befragten ihre wahren Entschädigungsforderungen bzw. ihre tatsächlichen 
Zahlungsbereitschaften, wenn sie erwarten, dass die Erhebung finanzielle oder politische Konsequenzen haben könnte.

Daher wurden die Haushalte nach Abgabe ihrer Entschädigungsforderung bzw. Zahlungsbereitschaft für Versorgungssicherheit gefragt, für wie wahrscheinlich sie es halten, dass sie ein solches Angebot tatsächlich erhalten und die Antworten somit finanzielle Konsequenzen haben könnten. Zusätzlich fragten wir nach einer Einschätzung, für wie wahrscheinlich die Haushalte es halten, dass die Erhebung politische Entscheidungen zur Versorgungssicherheit beeinflussen kann. Es gab jeweils fünf Antwortmöglichkeiten, die von sehr unwahrscheinlich bis sehr wahrscheinlich reichten. Laut Carson und Groves (2007) ist selbst ein geringer Glaube daran, dass die Befragungsergebnisse finanzielle oder politische Konsequenzen haben könnten, ausreichend, damit die Befragten ihre wahren Präferenzen offenbaren.

Tatsächlich ergeben sich für jene Haushalte, die es nicht für sehr unwahrscheinlich halten, dass die Befragungsergebnisse finanzielle Konsequenzen haben könnten, höhere Zustimmungsraten zu den vorgegebenen WTA- bzw. WTP-Beträgen als für jene, die nicht an finanzielle Konsequenzen glauben (siehe z. B. Mitani, Flores, 2014). Grob übereinstimmend mit den Ergebnissen von Herriges et al. (2010), Hwang et al. (2014) und Vossler und Watson (2013) fallen auch die Zustimmungsraten jener Haushalte, die politische Konsequenzen erwarten, geringfügig höher aus als für jene, die nicht an politische Konsequenzen glauben.

Probanden, welche die ihnen präsentierten WTA- bzw. WTP-Angebote ablehnten, wurden im Anschluss gefragt, ob sie, wenngleich nicht beim ihnen vorgelegten Angebot, so doch grundsätzlich bereit seien, Stromausfälle gegen eine Entschädigungszahlung zu akzeptieren, und falls ja, wie hoch diese ausfallen müsste. Mit einem Anteil von 24,5\% gab rund ein Viertel der das ihnen präsentierte Angebot Ablehnenden an (Tabelle 4), Stromausfälle gegen eine entsprechende Entschädigungszahlung akzeptieren zu wollen. Immerhin 10,2\% der „Ablehner“ der WTP-Angebote waren grundsätzlich zu einer Zahlung für Versorgungssicherheit bereit. Für die 384 Haushalte, die eine positive Zahlungsbereitschaft bekundeten, liegt der Median der Zahlungsbereitschaft für die Vermeidung eines unangekündigten, vierstündigen Stromausfalls bei 2 Euro pro Monat. 
Tabelle 4: Durchschnittliche Entschädigungsforderungen bzw. Zahlungsbereitschaften für diejenigen, die die ihnen präsentierten WTA- bzw. WTP-Angebote abgelehnt haben

\begin{tabular}{|c|c|c|c|c|c|c|}
\hline & \multicolumn{2}{|r|}{ WTA } & \multicolumn{4}{|c|}{ WTP } \\
\hline & $\begin{array}{l}\text { Anzahl der } \\
\text { Ablehnungen } \\
\text { mit positiven }\end{array}$ & $\begin{array}{c}\text { Anteil der } \\
\text { Ablehnungen } \\
\text { VTA-Werten }\end{array}$ & Median & $\begin{array}{l}\text { Anzahl der } \\
\text { Ablehnungen } \\
\text { mit positiven }\end{array}$ & $\begin{array}{l}\text { Anteil der } \\
\text { Ablehnungen } \\
\text { ГP-Werten }\end{array}$ & Median \\
\hline 3 Euro & 300 & $23,5 \%$ & 10,0 Euro & 125 & $11,0 \%$ & 1,0 Euro \\
\hline 6 Euro & 308 & $24,0 \%$ & 10,0 Euro & 124 & $9,7 \%$ & 2,0 Euro \\
\hline 9 Euro & 300 & $26,2 \%$ & 15,0 Euro & 135 & $9,9 \%$ & 3,0 Euro \\
\hline Insgesamt & 908 & $24,5 \%$ & 10,0 Euro & 384 & $10,2 \%$ & 2,0 Euro \\
\hline
\end{tabular}

Demgegenüber werden weitaus höhere Entschädigungsforderungen bei Stromausfällen gestellt: Der Median der Forderungen jener 908 Haushalte, die Stromausfälle gegen eine entsprechende Entschädigung akzeptieren würden, liegt bei 10 Euro. Somit ist ein gravierender Unterschied zwischen den durchschnittlichen Entschädigungsforderungen und Zahlungsbereitschaften zu konstatieren. Dieser übersteigt das von Schubert et al. (2013) und Praktiknjo (2014) gefundene WTA-WTP-Verhältnis von 3 deutlich. Dies ist umso bemerkenswerter, als unsere Studie im Gegensatz zu der von Schubert et al. (2013) auf einer deutschlandweiten Befragung beruht.

Tabelle 4 zeigt darüber hinaus, dass die Anteile der Haushalte, die das WTA- bzw. WTPAngebot ablehnen, nahezu unabhängig von der Höhe der Angebote ausfallen. Hingegen steigt der Median der Entschädigungsforderungen bzw. der Zahlungsbereitschaften mit der Höhe der angebotenen Beträge an. Beispielsweise liegt der Median der Entschädigungsforderungen von Haushalten, die eine ihnen offerierte Entschädigung von 9 Euro abgelehnt haben, bei 15 Euro und damit höher als bei jenen Haushalten, die Angebote über 3 bzw. 6. Euro abgelehnt haben. Es ist daher nicht auszuschließen, dass die Haushalte die ihnen offerierten Angebote als einen Fixpunkt ansehen, an dem sie sich orientieren. Diese Resultate bestätigen frühere empirische Ergebnisse zum sogenannten „Anker-Effekt“ (Tversky, Kahneman, 1974) in Bewertungsstudien (Green et al., 1998).

\section{Regressionsergebnisse}

Um zu ermitteln, welche individuellen Charakteristika höhere Entschädigungsforderungen für die Akzeptanz von Stromausfällen bzw. eine höhere Zahlungsbereitschaft für 
Versorgungssicherheit begünstigen, wird nun das folgende Lineare WahrscheinlichkeitsModell (LPM, Linear Probability Model) geschätzt: ${ }^{4}$

$$
\mathrm{y}_{\mathrm{i}}=\beta_{0}+\beta_{6} 6 \text { Euro }+\beta_{9} \text { 9Euro }+\boldsymbol{\beta}^{\mathrm{T}} \mathbf{x}_{\mathrm{i}}+\varepsilon_{\mathrm{i}} \text {. }
$$

Die abhängige Variable $y_{i}$ stellt einen binären Indikator dar, der den Wert 1 annimmt, falls die Frage zur Akzeptanz einer Entschädigung bzw. einer Zahlung von 3, 6 oder 9 Euro pro Monat mit „Ja“ beantwortet wird, andernfalls den Wert 0. Geforderte Zahlungsbeträge bzw. angebotene hypothetische Entschädigungen in Höhe von 6 bzw. 9 Euro werden durch die entsprechenden Indikatorvariablen wiedergegeben. Der Vektor $\mathbf{x}$ umfasst alle übrigen Variablen, insbesondere die sozio-ökonomischen Charakteristika.

Die in Tabelle 5 dargestellten Schätzergebnisse bestätigen die deskriptiven Resultate aus Tabelle 3. So steigt die Akzeptanz von Entschädigungen grundsätzlich mit zunehmender Höhe der WTA-Beträge. Die Bereitschaft, einen vorgegebenen Betrag zu bezahlen, sinkt hingegen mit wachsenden WTP-Werten. Mit wenigen Ausnahmen zeigen sich bei den sozioökonomischen Charakteristika keine statistisch signifikanten Effekte. Auswirkungen hat es jedoch, wenn Kinder im Haushalt leben. Dies senkt die Zahlungsbereitschaft für Versorgungssicherheit.

Es überrascht, dass das Einkommen und der Stromverbrauch der Haushalte sowie deren Erfahrung mit Stromausfällen von mindestens halbstündiger Dauer keinerlei Einfluss haben, weder auf die Akzeptanz von Entschädigungsforderungen noch auf die Bereitschaft, für Versorgungsicherheit zu bezahlen. Das letztere Resultat steht im Kontrast zu den üblichen Ergebnissen der empirischen Literatur. So finden zum Beispiel Carlsson et al. (2011), dass die Zahlungsbereitschaft für Versorgungssicherheit nach der Erfahrung mit einem Stromausfall stark ansteigt.

Allerdings dürfte in diesem Beispiel die Erfahrung mit einem Stromausfall nach einem schweren Sturm in Schweden noch sehr lange nachgewirkt haben und war den schwedischen Haushalten zum Zeitpunkt der Erhebung vermutlich noch sehr präsent. Dies stünde in Einklang mit der sogenannten „Availability Heuristic“ (Tversky, Kahneman, 1973). Angewandt auf das vorliegende empirische Beispiel bezieht sich diese Art von

\footnotetext{
${ }^{4}$ Im Gegensatz zu nichtlinearen Modellen ignoriert das LPM die Binarität der abhängigen Variablen und fasst diese als stetige Variable auf. Dies kann zu Verzerrungen führen. Allerdings plädieren Angrist und Pischke (2009) bei unbekannter Verteilung der Daten für die Nutzung des LPM, anstatt z. B. eines LogitModells, wie es hier zusätzlich geschätzt wurde. Die im Anhang dargestellten marginalen Effekte für das entsprechende Logit-Modell sind qualitativ und quantitativ sehr ähnlich zu den Schätzwerten für das LPM (Tabellen A2 - A4).
} 
Faustregel auf die Leichtigkeit, mit der sich die Befragten an Stromausfälle erinnern können, wenn sie nach einer Einschätzung der Wahrscheinlichkeit von zukünftigen Stromausfällen sowie nach ihrer Zahlungsbereitschaft für deren Vermeidung gefragt werden.

Tabelle 5: Schätzergebnisse für das Lineare Wahrscheinlichkeitsmodell zur Modellierung der Zustimmung zu vorgegebenen WTA- bzw. WTP-Werten

\begin{tabular}{lcccc}
\hline & \multicolumn{2}{c}{ WTA } & \multicolumn{2}{c}{ WTP } \\
\cline { 2 - 5 } & Koeff. & Std. Fehler & Koeff. & Std. Fehler \\
\hline 6 Euro & $0,054^{*}$ & $(0,025)$ & $-0,083^{* *}$ & $(0,023)$ \\
9 Euro & $0,071^{* *}$ & $(0,026)$ & $-0,094^{* *}$ & $(0,023)$ \\
Alter & $-0,013^{*}$ & $(0,006)$ & $-0,001$ & $(0,005)$ \\
Alter x Alter & $0,0001^{*}$ & $(0,0001)$ & 0,00001 & $(0,00005)$ \\
Frau & 0,020 & $(0,024)$ & 0,024 & $(0,021)$ \\
Kinder im Haushalt & 0,017 & $(0,035)$ & $-0,074^{*}$ & $(0,031)$ \\
Hochschulabschluss & $0,054^{* *}$ & $(0,023)$ & 0,021 & $(0,021)$ \\
ln(Einkommen) & $-0,030$ & $(0,036)$ & 0,012 & $(0,031)$ \\
Grünen-Wähler & 0,038 & $(0,036)$ & $-0,005$ & $(0,034)$ \\
ln(Jährlicher Stromverbrauch) & $-0,014$ & $(0,013)$ & $-0,008$ & $(0,012)$ \\
Stromausfall erlebt & $-0,020$ & $(0,022)$ & $-0,014$ & $(0,020)$ \\
Konstante & $0,813^{* *}$ & $(0,326)$ & 0,201 & $(0,278)$ \\
\hline Anzahl an Beobachtungen: & \multicolumn{2}{c}{1.469} & \multicolumn{3}{c}{1.512} \\
\hline
\end{tabular}

Anmerkung: Standardfehler in Klammern. ** bzw.* zeigen statistische Signifikanz auf dem 1\%- bzw. 5\%Niveau an.

Sehr ähnliche Ergebnisse wie die in Tabelle 5 dargestellten erhalten wir für jene Befragten, die einen mehr oder weniger großen Glauben daran besitzen, dass die Befragung finanzielle Konsequenzen für sie haben könnte, etwa weil ihr Stromanbieter tatsächlich mit entsprechenden Angeboten auf sie zukommen könnte (Tabelle 6). Wie in den Tabellen 3 und 5 zeigt sich auch in diesem Fall, dass höhere Entschädigungsangebote eher akzeptiert werden und die Bereitschaft, monatliche Zahlungen für Versorgungssicherheit zu leisten, mit wachsenden Beträgen abnimmt. Mit Ausnahme vom negativen Effekt, den die Existenz von Kindern im Haushalt auf die Zahlungsbereitschaft für Versorgungssicherheit hat, zeigt keine der übrigen erklärenden Variablen bei den üblichen Signifikanzniveaus keine einen statistisch signifikanten Einfluss. 
Tabelle 6: LPM-Schätzergebnisse für die Zustimmung zu vorgegebenen WTA- bzw. WTP-Werten für Haushalte, die finanzielle Konsequenzen erwarten

\begin{tabular}{lcccc}
\hline & \multicolumn{2}{c}{ WTA } & \multicolumn{2}{c}{ WTP } \\
\cline { 2 - 5 } & Koeff. & Std. Fehler & Koeff. & Std. Fehler \\
\hline 6 Euro & $0,109^{*}$ & $(0,058)$ & $-0,136^{* *}$ & $(0,047)$ \\
9 Euro & $0,139^{* *}$ & $(0,060)$ & $-0,135^{* *}$ & $(0,048)$ \\
Alter & $-0,006$ & $(0,011)$ & $-0,005$ & $(0,010)$ \\
Alter x Alter & 0,0001 & $(0,0001)$ & 0,00001 & $(0,0001)$ \\
Frau & 0,039 & $(0,057)$ & 0,048 & $(0,043)$ \\
Kinder im Haushalt & 0,034 & $(0,082)$ & $-0,127^{* *}$ & $(0,064)$ \\
Hochschulabschluss & 0,070 & $(0,052)$ & $-0,010$ & $(0,042)$ \\
ln(Einkommen) & $-0,083$ & $(0,078)$ & 0,028 & $(0,060)$ \\
Grünen-Wähler & 0,078 & $(0,079)$ & $-0,016$ & $(0,065)$ \\
ln(Jährlicher Stromverbrauch) & $-0,008$ & $(0,030)$ & 0,028 & $(0,027)$ \\
Stromausfall erlebt & $-0,028$ & $(0,049)$ & $-0,008$ & $(0,041)$ \\
Konstante & 1,039 & $(0,671)$ & $-0,016$ & $(0,510)$ \\
\hline Anzahl an Beobachtungen: & \multicolumn{3}{c}{438} \\
\hline
\end{tabular}

Anmerkung: Standardfehler in Klammern. ${ }^{* *}$ bzw. ${ }^{*}$ zeigen statistische Signifikanz auf dem 1\%- bzw. 5\%Niveau an.

Bei Haushalten, die politische Konsequenzen erwarten (Tabelle 7), ergibt sich für die Akzeptanz von monatlichen Zahlungen für Versorgungssicherheit ein ähnliches Bild wie in den Tabellen 5 und 6. Allein bei den Entschädigungsforderungen gibt es im Vergleich zur gesamten Stichprobe einen Unterschied: Entschädigungen werden von Frauen eher akzeptiert als von Männern.

Tabelle 7: LPM-Schätzergebnisse für die Zustimmung zu vorgegebenen WTA- bzw. WTP-Werten für Haushalte, die politische Konsequenzen erwarten

\begin{tabular}{lcccc}
\hline & \multicolumn{2}{c}{ WTA } & \multicolumn{2}{c}{ WTP } \\
\cline { 2 - 5 } & Koeff. & Std. Fehler & Koeff. & Std. Fehler \\
\hline 6 Euro & $0,062^{* *}$ & $(0,030)$ & $-0,077^{* *}$ & $(0,028)$ \\
9 Euro & $0,096^{* *}$ & $(0,030)$ & $-0,088^{* *}$ & $(0,027)$ \\
Alter & $-0,013^{*}$ & $(0,063)$ & $-0,007$ & $(0,006)$ \\
Alter x Alter & $0,0001^{*}$ & $(0,0001)$ & 0,00001 & $(0,0001)$ \\
Frau & $0,062^{*}$ & $(0,029)$ & 0,015 & $(0,026)$ \\
Kinder im Haushalt & $-0,046$ & $(0,042)$ & $-0,082^{*}$ & $(0,037)$ \\
Hochschulabschluss & $0,061^{*}$ & $(0,061)$ & $-0,032$ & $(0,025)$ \\
ln(Einkommen) & $-0,059$ & $(0,017)$ & 0,008 & $(0,037)$ \\
Grünen-Wähler & 0,056 & $(0,041)$ & $-0,001$ & $(0,038)$ \\
ln(Jährlicher Stromverbrauch) & $-0,021$ & $(0,017)$ & 0,001 & $(0,014)$ \\
Stromausfall erlebt & $-0,046$ & $(0,026)$ & $-0,008$ & $(0,024)$ \\
Konstante & 1,112 & $(0,387)$ & $-0,003$ & $(0,332)$ \\
\hline Anzahl an Beobachtungen: & \multicolumn{2}{c}{1.042} & & 1.078
\end{tabular}

Anmerkung: Standardfehler in Klammern. ** bzw.* zeigen statistische Signifikanz auf dem 1\%- bzw. 5\%Niveau an. 


\section{Zusammenfassung und Schlussfolgerung}

Die Versorgungssicherheit mit Strom ist in Deutschland eine Selbstverständlichkeit - im Gegensatz zu vielen anderen Ländern, einschließlich den USA, wo die Kraftwerks- und Netzinfrastruktur weit weniger gut ausgebaut ist als in Deutschland. Als Folge der Energiewende und der zunehmenden Stilllegung konventioneller Kraftwerke, insbesondere der Atomkraftwerke, sowie des massiven Ausbaus der volatilen Stromerzeugung auf Basis von regenerativen Stromerzeugungstechnologien, vor allem der Windkraft und Photovoltaik, könnte sich in Ermangelung ausreichender Stromspeicherkapazitäten die Versorgungssicherheit künftig verschlechtern. Tatsächlich müssen die Betreiber der großen Übertragungsnetze, die wesentlich für die Aufrechterhaltung der Netzstabilität in Deutschland verantwortlich sind, zu diesem Zweck heutzutage mehrmals täglich in den Netzbetrieb eingreifen, während dies in der Vergangenheit lediglich wenige Male im Jahr vorkam. Damit verbunden ist das wachsende Risiko eines großflächigen Stromausfalls.

Vor diesem Hintergrund hat dieser Beitrag auf Basis einer Befragung von mehr als 5.000 Haushaltsvorständen untersucht, wie viel sie für Versorgungssicherheit mit Strom zu zahlen bereit sind. Alternativ zur Zahlungsbereitschaft (willingness to pay, WTP) wurde auch nach der Bereitschaft gefragt, gegen eine Entschädigungszahlung auf ein gewisses $\mathrm{Ma}$ an Versorgungssicherheit zu verzichten (willingness to accept, WTA).

In Übereinstimmung mit zahlreichen Studien der empirischen Literatur finden wir mittlere WTA-Werte für Entschädigungsforderungen, die deutlich über den mittleren WTPWerten für die Zahlungsbereitschaft für die Vermeidung eines unangekündigten, vierstündigen Stromausfalls liegen. Den Grund für diese Diskrepanz sehen wir darin, dass die bekundeten Entschädigungsforderungen für den Verzicht auf Versorgungssicherheit aus strategischen Gründen tendenziell über dem tatsächlichen Wert liegen, der der Versorgungssicherheit mit Strom beigemessen wird, wohingegen die dafür bekundete Zahlungsbereitschaft tendenziell untertrieben wird. Für Stromversorger, die ihren Kunden künftig Verträge anbieten möchten, in denen Versorgungssicherheit eine Rolle spielt, wäre es nach diesen Ergebnissen wenig ratsam, hohe Entschädigungszahlungen für die Möglichkeit anzubieten, unangekündigte Stromabschaltungen vornehmen zu dürfen.

Angesichts der WTA-WTP-Lücke, die in diesem Beitrag im Zusammenhang mit Stromversorgungssicherheit gefunden wurde, und der von uns angeführten Erklärung für 
diese Lücke ist für die künftige Forschung der Schluss zu ziehen, dass immer versucht werden sollte, beide Präferenzmaße abzufragen, die Zahlungsbereitschaft und die Entschädigungsforderung, da die wahre Zahlungsbereitschaft aufgrund strategischen Verhaltens vermutlich zwischen den angegebenen WTA- und WTP-Werten liegen wird. Dies gilt insbesondere für das - bislang als so selbstverständlich erachtete - Gut der Versorgungssicherheit, für das die für eine gute Einschätzung ihres Wertes nötige Erfahrung nahezu gänzlich fehlt. 


\section{Appendix}

\section{Experiment zur Versorgungssicherheit: Die Fragen}

Aus Gründen der Versorgungssicherheit kann es manchmal sinnvoll sein, einzelnen Haushalten vorübergehend den Strom abzustellen.

Frage WTA-Gruppe: Stellen Sie sich vor, Ihr lokaler Netzbetreiber bietet Ihnen daher eine monatliche Entschädigung in Höhe von WTA $[\in\{3,6,9\}]$ Euro (d. h. WTA*12 Euro im Jahr) dafür an, Ihnen den Strom einmal im Jahr für maximal 4 Stunden unangekündigt abstellen zu dürfen. Würden Sie dieses Angebot annehmen?

Frage WTP-Gruppe: Stellen Sie sich vor, Ihr lokaler Netzbetreiber fragt Sie daher, ob Sie bereit sind, WTP $[\in\{3,6,9\}]$ Euro monatlich (d. h. WTP*12 Euro im Jahr) zu zahlen, damit Ihnen der Strom nicht abgestellt wird. Der Netzbetreiber dürfte Ihnen ansonsten einmal im Jahr für maximal 4 Stunden unangekündigt den Strom abstellen. Würden Sie dieses Angebot annehmen?

Die folgende Frage wurde nur Haushalten gestellt, welche die oben genannte Frage der WTA-Gruppe mit „nein“ oder „weiß nicht“ beantwortet haben.

\section{Frage nach der grundsätzlichen Bereitschaft zur Akzeptanz einer Entschädigung:}

Wären Sie grundsätzlich bereit, zu akzeptieren, dass man Ihnen aus Gründen der Versorgungssicherheit den Strom einmal im Jahr abstellt, wenn Sie im Gegenzug dafür eine Entschädigung erhalten würden?

Die folgende Frage wurde nur Haushalten gestellt, welche die vorige Frage mit ,ja“ beantwortet haben.

Entschädigung: Wie hoch müsste die Entschädigung sein, damit Sie akzeptieren würden, dass Ihnen einmal im Jahr der Strom unangekündigt für maximal 4 Stunden abgestellt wird? Stellen Sie sich vor, Sie würden eine monatliche Entschädigung erhalten. Bitte runden Sie ihre Angabe auf volle Euro.

Die folgende Frage wurde nur Haushalten gestellt, welche die oben genannte Frage der WTP-Gruppe mit „nein“ oder „,weiß nicht“ beantwortet haben. 
Frage grundsätzliche Zahlungsbereitschaft: Wären Sie grundsätzlich zu einer Zusatzzahlung bereit, um $\mathrm{zu}$ verhindern, dass Ihnen aus Gründen der Versorgungssicherheit einmal im Jahr der Strom abgestellt werden dürfte?

Die folgende Frage wurde nur Haushalten gestellt, welche die vorige Frage mit ,ja“ beantwortet haben.

Zusatz-Zahlung: Wie viel wären Sie maximal bereit, zusätzlich pro Monat zu zahlen, damit Ihnen nicht einmal im Jahr unangekündigt für maximal 4 Stunden der Strom abgestellt werden darf?

Frage finanzielle Konsequenzen: Für wie wahrscheinlich halten Sie es, dass Ihr Stromversorger tatsächlich mit einem solchen Angebot auf Sie zukommen wird?

Frage politische Konsequenzen: Für wie wahrscheinlich halten Sie es, dass die Ergebnisse von Befragungen wie dieser die politischen Entscheidungen zur Versorgungssicherheit beeinflussen?

\section{Tabellen-Anhang}

Tabelle A1: Vergleich unserer Stichprobe mit der Grundgesamtheit an deutschen Haushalten

\begin{tabular}{lcc}
\hline Variable & Stichprobe & Grundgesamtheit \\
\hline Alter unter 25 Jahren & $1,1 \%$ & $4,6 \%$ \\
Alter 25 - 64 Jahre & $72,0 \%$ & $67,0 \%$ \\
Alter 65 Jahre und mehr & $26,9 \%$ & $28,4 \%$ \\
Frau & $37,9 \%$ & $35,5 \%$ \\
Ostdeutschland & $20,0 \%$ & $20,9 \%$ \\
Hochschulabschluss & $33,0 \%$ & $20,4 \%$ \\
Haushaltsnettoeinkommen $\geq 4.700$ Euro & $11,9 \%$ & $11,4 \%$ \\
\hline
\end{tabular}

Anmerkung: Die Daten der Grundgesamtheit entstammen Destatis (2016). Das Statistische Bundesamt bittet den Haupteinkommensbezieher, den Fragebogen auszufüllen, während wir den Haushaltsvorstand befragen. Beim Statistischen Bundesamt Destatis (2016) ist das Haushaltsnettoeinkommen im Gegensatz zu unserer Erhebung bei 4.500 Euro top-codiert. 
Tabelle A2: Marginale Effekte des Logit-Modells zur Modellierung der Zustimmung zu vorgegebenen WTA- bzw. WTP-Werten

\begin{tabular}{lcccc}
\hline & \multicolumn{2}{c}{ WTA } & \multicolumn{2}{c}{ WTP } \\
\cline { 2 - 5 } & Koeff. & Std. Fehler & Koeff. & Std. Fehler \\
\hline 6 Euro & $0,053^{* *}$ & $(0,024)$ & $-0,083^{* * *}$ & $(0,024)$ \\
9 Euro & $0,071^{* * *}$ & $(0,025)$ & $-0,094^{* * *}$ & $(0,023)$ \\
Alter & $-0,000$ & $(0,001)$ & $-0,001$ & $(0,001)$ \\
Frau & 0,019 & $(0,024)$ & 0,026 & $(0,022)$ \\
Kinder im Haushalt & 0,019 & $(0,034)$ & $-0,072 * *$ & $(0,032)$ \\
Hochschulabschluss & $0,054 * *$ & $(0,024)$ & 0,020 & $(0,021)$ \\
ln(Einkommen) & $-0,027$ & $(0,035)$ & 0,015 & $(0,033)$ \\
Grünen-Wähler & 0,038 & $(0,037)$ & $-0,005$ & $(0,033)$ \\
ln(Jährlicher Stromverbrauch) & $-0,012$ & $(0,012)$ & $-0,008$ & $(0,011)$ \\
Stromausfall erlebt & $-0,021$ & $(0,021)$ & $-0,014$ & $(0,019)$ \\
\hline Anzahl an Beobachtungen & \multicolumn{2}{c}{1.469} & \multicolumn{2}{c}{1.512}
\end{tabular}

Anmerkung: Standardfehler in Klammern. ${ }^{* *}$ bzw.* zeigen statistische Signifikanz auf dem 1\%- bzw. 5\%Niveau an.

Tabelle A3: Marginale Effekte des Logit-Modells für die Zustimmung zu vorgegebenen WTA- bzw. WTPWerten für Haushalte, die finanzielle Konsequenzen erwarten

\begin{tabular}{lcccc}
\hline & \multicolumn{2}{c}{ WTA } & \multicolumn{2}{c}{ WTP } \\
\cline { 2 - 5 } & Koeff. & Std. Fehler & Koeff. & Std. Fehler \\
\hline 6 Euro & $0,108^{*}$ & $(0,055)$ & $-0,135^{* * *}$ & $(0,047)$ \\
9 Euro & $0,138^{* *}$ & $(0,057)$ & $-0,137^{* * *}$ & $(0,048)$ \\
Alter & 0,001 & $(0,002)$ & $0,003^{*}$ & $(0,002)$ \\
Frau & 0,037 & $(0,058)$ & 0,053 & $(0,045)$ \\
Kinder im Haushalt & 0,034 & $(0,081)$ & $-0,118^{*}$ & $(0,067)$ \\
Hochschulabschluss & 0,067 & $(0,052)$ & $-0,009$ & $(0,040)$ \\
ln(Einkommen) & $-0,078$ & $(0,075)$ & 0,037 & $(0,065)$ \\
Grünen-Wähler & 0,079 & $(0,082)$ & $-0,020$ & $(0,065)$ \\
ln(Jährlicher Stromverbrauch) & $-0,008$ & $(0,030)$ & 0,029 & $(0,027)$ \\
Stromausfall erlebt & $-0,028$ & $(0,048)$ & $-0,008$ & $(0,040)$ \\
\hline Anzahl an Beobachtungen & \multicolumn{3}{c}{438} \\
\hline
\end{tabular}

Anmerkung: Standardfehler in Klammern. ** bzw.* zeigen statistische Signifikanz auf dem 1\%- bzw. 5\%Niveau an.

Tabelle A4: Marginale Effekte des Logit-Modells für die Zustimmung zu vorgegebenen WTA- bzw. WTPWerten für Haushalte, die politische Konsequenzen erwarten

\begin{tabular}{lcccc}
\hline & \multicolumn{2}{c}{ WTA } & \multicolumn{2}{c}{ WTP } \\
\cline { 2 - 5 } & Koeff. & Std. Fehler & Koeff. & Std. Fehler \\
\hline 6 Euro & $0,061^{* *}$ & $(0,029)$ & $-0,077^{* * *}$ & $(0,028)$ \\
9 Euro & $0,096^{* * *}$ & $(0,030)$ & $-0,087^{* * *}$ & $(0,028)$ \\
Alter & 0,0004 & $(0,001)$ & 0,001 & $(0,001)$ \\
Frau & $0,061^{* *}$ & $(0,031)$ & 0,017 & $(0,026)$ \\
Kinder im Haushalt & $-0,046$ & $(0,041)$ & $-0,081^{* *}$ & $(0,038)$ \\
Hochschulabschluss & $0,060^{* *}$ & $(0,028)$ & 0,031 & $(0,025)$ \\
ln(Einkommen) & $-0,057$ & $(0,042)$ & 0,01 & $(0,039)$ \\
Grünen-Wähler & 0,052 & $(0,043)$ & $-0,001$ & $(0,038)$ \\
ln(Jährlicher Stromverbrauch) & $-0,021$ & $(0,016)$ & $-0,001$ & $(0,014)$ \\
Stromausfall erlebt & $-0,046^{*}$ & $(0,025)$ & $-0,008$ & $(0,023)$ \\
\hline Anzahl an Beobachtungen & \multicolumn{2}{c}{1.042} & \multicolumn{2}{c}{1.078}
\end{tabular}

Anmerkung: Standardfehler in Klammern. ** bzw. ${ }^{*}$ zeigen statistische Signifikanz auf dem 1\%- bzw. 5\%Niveau an. 


\section{Literatur}

Amigues, Jean-Pierre, Catherine Boulatoff, Brigitte Desaigues, Caroline Gauthier, John E. Keith (2002). „The benefits and costs of riparian analysis habitat preservation: A willingness to accept/willingnesss to pay contingent valuation approach", Ecological Economics 43 (1), 17-31.

Andor, Mark, Manuel Frondel, Colin Vance (2014), „Hypothetische Zahlungsbereitschaft für grünen Strom: Bekundete Präferenzen privater Haushalte für das Jahr 2013“, Perspektiven der Wirtschaftspolitik 15 (4), 355-366.

Angrist, Joshua. D., Jörn-Steffen Pischke (2009), Mostly harmless econometrics: An empiricist's companion. Princeton University Press.

Arrow, Kenneth, Robert Solow, Paul R. Portney, Edward E. Leamer, Roy Radner, Howard Schuman (1993), „Report of the NOAA panel on contingent valuation”, Federal Register 58 (10), 4601-4614.

BDEW (2017), Redispatch in Deutschland. Auswertung der Transparenzdaten. April 2013 bis einschließlich März 2017. Bundesverband der Energie- und Wasserwirtschaft, Berlin.

Blumenschein, Karen, Glenn C. Blomquist, Magnus Johannesson, Nancy Horn, Patricia Freeman (2008), „Eliciting willingness to pay without bias: Evidence from a field experiment", Economic Journal 118 (525), 114-137.

BMWi (2017), Zeitreihen zur Entwicklung der erneuerbaren Energien in Deutschland. Bundesministerium für Wirtschaft und Energie, Berlin.

BNetzA (2017), Versorgungsqualität - SAIDI-Werte 2006-2015. Bundesnetzagentur, Bonn. URL:

https://www.bundesnetzagentur.de/DE/Sachgebiete/ElektrizitaetundGas/Unternehmen_Ins titutionen/Versorgungssicherheit/Versorgungsunterbrechungen/Auswertung_Strom/Verso rqungsunterbrech_Strom_node.html. Abgerufen am 22. August 2017.

Carlsson, Frederik, Peter Martinsson, Alpaslan Akay (2011), „The effect of power outages and cheap talk on willingness to pay to reduce outages", Energy Economics 33 (5), 790 798. 
Carson, Richard T., Theodore Groves (2007), „Incentive and informational properties of preference questions", Environmental and Resource Economics 37 (1), 181-210.

CEER (2016), 6th CEER benchmarking report on the quality of electricity and gas supply. Council of European Energy Regulators, Brüssel.

Destatis (2016), Bevölkerung und Erwerbstätigkeit. Haushalte und Familien. Ergebnisse des Mikrozensus. Artikelnummer: 2010300157004. Statistisches Bundesamt, Wiesbaden.

Green, Donald, Karen E. Jacowitz, Daniel Kahneman, Daniel McFadden (1998), „Referendum contingent valuation, anchoring, and willingness to pay for public goods”, Resource and Energy Economics, 20 (2), 85-116.

Groothuis, Peter A., Gail Miller (1994), „Locating hazardous waste facilities: The influence of NIMBY beliefs", American Journal of Economics and Sociology 53 (3), 335346.

Growitsch, Christian, Raimund Malischek, Sebastian Nick, Heike Wetzel (2015), „The costs of power interruptions in Germany: A regional and sectoral analysis", German Economic Review 16 (3), 307-323.

Guria, Jagadish, Joanne Leung, Michael Jones-Lee, Graham Loomes (2005), „The willingness to accept value of statistical life relative to the willingness to pay value: Evidence and policy implications", Environmental and Resource Economics 32 (1), 113127.

Hanemann, Michael W. (1991), „Willingness to pay and willingness to accept: How much can they differ?", American Economic Review 81 (3), 635-647.

Harrison, Glenn W. (2006), „Experimental evidence on alternative environmental valuation methods", Environmental and Resource Economics 34 (1), 125-162.

Herriges, Joseph, Catherine Kling, Chih-Chen Liu, Justin Tobias (2010), „What are the consequences of consequentiality? Journal of Environmental Economics and Management 59 (1), 67-81.

Hochloff, Patrick, Benedikt Meyer, Jan von Appen et al. (2014), „Metastudie Energiespeicher", Studie im Auftrag des Bundesministeriums für Wirtschaft und Energie (BMWi), Abschlussbericht. Fraunhofer-Institut für Umwelt-, Sicherheits- und 
Energietechnik UMSICHT und Fraunhofer-Institut für Windenergie und Energiesystemtechnik IWES, Oberhausen / Kassel.

Horowitz, John K., Kenneth E. McConnell (2002), „A review of WTA/WTP studies”, Journal of Environmental Economics and Management 44 (3), 426-447.

Hwang, Joonghyun, Daniel R. Petrolia, Matthew G. Interis (2014), „Consequentiality and opt-out responses in stated preference surveys", Agricultural and Resource Economics Review 43 (3), 471-488.

Isoni, Andrea, Graham Loomes, Robert Sugden (2011), „The willingness to paywillingness to accept gap, the 'endowment effect' subject misconceptions, and experimental procedures for eliciting valuations: Comment", American Economic Review $101(2), 991-1011$.

Kahneman, Daniel, Jack L. Knetsch, Richard H. Thaler (1990), „Experimental tests of the endowment effect and the Coase theorem", Journal of Political Economy 98 (6), 13251348.

Knetsch, Jack. L. (1990), „Environmental policy implications of disparities between willingness to pay and compensation demanded measures of values", Journal of Environmental Economics and Management 18 (3), 227-237.

Koetse, Mark J., Roy Brouwer (2016), „Reference dependence effects on WTA and WTP value functions and their disparity", Environmental and Resource Economics 65 (4), 723 745.

List, John A., Craig A. Gallet (2001), „What experimental protocol influence disparities between actual and hypothetical stated values? Environmental and Resource Economics 20 (3), 241-254.

Loomis, John, Cynthia Pierce, Mike Manfredo (2000), „Using the demand for hunting licenses to evaluate contingent valuation estimates of willingness to pay", Applied Economics Letters 7 (7),435-438.

Mandler, Michael (2004), „Status quo maintenance reconsidered: Changing or incomplete preferences?”, Economic Journal 114 (499), F518-F535. 
Mitani, Yohei, Nicholas E. Flores (2014), „Hypothetical bias reconsidered: Payment and provision uncertainties in a threshold provision mechanism", Environmental and Resource Economics 59 (3), 433-454.

Nape, Steven, Peter Frykblom, Glenn W. Harrison, James C. Lesley, (2003), „Hypothetical bias and willingness to accept”, Economics Letters 78 (3), 423-430.

Plott, Charles R., Kathryn Zeiler (2005), „The willingness to pay-willingness to accept gap", American Economic Review 95 (3), 530-545.

Plott, Charles R., Kathryn Zeiler (2011), „The willingness to pay-willingness to accept gap, the 'endowment effect', subject misconceptions, and experimental procedures for eliciting valuations: Reply”, American Economic Review 101 (2), 1012-1028.

Praktiknjo, Aaron J. (2014), „Stated preferences based estimation of power interruption costs in private households: An example from Germany", Energy 76, 82-90.

Praktiknjo, Aaron J., Alexander Hähnel, Georg Erdmann (2011), „Assessing energy supply security: Outage costs in private households”, Energy Policy 39 (12), 7825-7833.

Römer, Benedikt, Philipp Reichhart, Johann Kranz, Arnold Picot (2012), „The role of smart metering and decentralized electricity storage for smart grids: The importance of positive externalities”, Energy Policy 50, 486-495.

Röpke, Luise (2013), „The development of renewable energies and supply security: A trade-off analysis", Energy Policy 61, 1011-1021.

Schubert, Daniel K. J., Alexander von Selasinsky, Thomas Meyer, Adriane Schmidt, Sebastian Thuß, Niels Erdmann, Mark Erndt, Dominik Möst (2013), „Gefährden Stromausfälle die Energiewende? Einfluss auf Akzeptanz und Zahlungsbereitschaft“", Energiewirtschaftliche Tagesfragen 63 (10), 35-39.

Shogren, Jason F., Seung Y. Shin, Dermit J. Hayes, James B. Kliebenstein (1994), „Resolving differences in willingness to pay and willingness to accept“, American Economic Review 84 (1), 255-270.

Stenzel, Peter, Richard Bongartz, Ewgenji Kossi (2013), „Potenzialanalyse für Pumpspeicher an Bundeswasserstraßen in Deutschland“, Energiewirtschaftliche Tagesfragen 63 (3), 54-57. 
Thaler, Richard H. (1980), „Toward a positive theory of consumer choice”, Journal of Economic Behavior\& Organization 1 (1), 39-60.

Tunçel, Tuba, James K. Hammitt (2014), „A new meta-analysis on the WTP/WTA disparity”, Journal of Environmental Economics and Management 68 (1), 175-187.

Tversky, Amos, Daniel Kahneman (1973), „Availability: A heuristic for judging frequency and probability", Cognitive Psychology 5 (2), 207-232.

Tversky, Amos, Daniel Kahneman (1974), „Judgment under uncertainty: Heuristics and biases", Science 185, 1124-1131.

Vossler, Christian A., Maurice Doyon, Daniel Rondeau (2012), „Truth in consequentiality: Theory and field evidence on discrete choice experiments", American Economic Journal: Microeconomics 4 (4), 145-171.

Vossler, Christian A., Sharon B. Watson (2013), „Understanding the consequences of consequentiality: Testing the validity of stated preferences in the field", Journal of Economic Behavior \& Organization 86, 137-147.

Welt (2016), „EU will Deutschland in zwei Strompreiszonen teilen.“ URL: https://www.welt.de/wirtschaft/article155773887/EU-will-Deutschland-in-Zwei-

Strompreiszonen-teilen.html. Abgerufen am 22. August 2017.

Willig, Robert D. (1976), „Consumer's surplus without apology”, American Economic Review 66 (4), 589-597.

Wolf, André, Lars Wenzel (2016), „Regional diversity in the costs of electricity outages: Results for German counties", Utilities Policy 43, 195-205. 

